\title{
Meta-Analysis of the Efficacy of Virtual Reality Exposure Therapy for Social Anxiety
}

\author{
Rachel K. Chesham, John M. Malouff ${ }^{\star}$ and Nicola S. Schutte \\ University of New England, Armidale, New South Wales, Australia \\ ${ }^{\star}$ Corresponding author. John M. Malouff, University of New England, School of Psychology, Armidale NSW 2351, Australia. \\ Email: jmalouff@une.edu.au
}

\begin{abstract}
Social anxiety is a common, debilitating psychological problem. In the present study, two meta-analyses examined the efficacy of virtual reality exposure therapy for social anxiety. The first meta-analysis tested whether virtual reality exposure therapy reduces social anxiety more than a waitlist control condition. The results of the first meta-analysis, consisting of six studies and 233 participants, showed a significant overall effect size, indicating that virtual reality exposure therapy was effective in reducing social anxiety. The second meta-analysis tested whether the standard treatment for social anxiety, which includes in vivo or imaginal exposure, leads to greater effects than virtual reality exposure therapy. The second meta-analysis, consisting of seven studies and 340 total participants, showed essentially no difference in effect sizes between virtual reality exposure and in vivo or imaginal exposure. The results of the two meta-analyses support the use of virtual reality in the treatment of social anxiety.
\end{abstract}

Keywords: exposure therapy; meta-analysis; social anxiety; virtual reality

Social anxiety is characterised by fear of social situations and of interactions with others. Individuals experiencing social anxiety frequently avoid social situations due to fear of public scrutiny and negative evaluation (Hofmann \& DiBartolo, 2014). Research findings indicate that public speaking is the most prevalent social fear (Furmark, 2002; Ruscio et al., 2008). Other common social fears include interacting with strangers, dating, and going to parties (Hofmann, Heinrichs, \& Moscovitch, 2004). Research findings show that these fears can cause impairments in social functioning and are associated with lower levels of educational attainment, higher risk of unemployment, and difficulties in forming intimate relationships (Crome et al., 2015; Fehm, Pelissolo, Furmark, \& Wittchen, 2005).

Despite the various harmful impacts of high social anxiety, few individuals with social anxiety seek professional help (Crome et al., 2015; Fehm et al., 2005). One possible reason for the low rate of help seeking might be that social anxiety leads these individuals to avoid interactions with mental health care providers, just as they avoid interactions with others.

Socially anxious individuals may meet criteria for a diagnosis of social anxiety disorder, as described by the Diagnostic and Statistical Manual of Mental Disorders (DSM-5; American Psychiatric Association, 2013). According to the DSM-5, a diagnosis of social anxiety disorder (also known as social phobia) reflects clinically significant levels of social anxiety. Furthermore, the DSM-5 includes a 'performance only' specifier for individuals fearing only public speaking or performance situations.

Anxiety in social situations is a common human experience (Ruscio, 2010). Therefore, some experts argue it is best to conceptualise social anxiety as existing along a continuum ranging from fears that are adaptive to those that cause substantial impairment (Hofmann \& DiBartolo, 2014). Ruscio (2010) suggested that all levels of social anxiety are important to consider and that researchers may overlook important information by excluding milder cases that fall below the diagnostic threshold. 


\section{Theoretical and Treatment Models of Social Anxiety}

Cognitive behaviour therapy (CBT) is the first-line treatment for social anxiety (Pilling et al., 2013). While the main evidence-based CBT tools for social anxiety include exposure, cognitive restructuring, social skills training and relaxation training, standard treatment almost always includes exposure as a key component (Heimberg, 2002).

The therapist and the client typically generate a hierarchy of social situations to use in gradual in vivo or imaginal exposure (Deacon \& Abramowitz, 2004; Rodebaugh, Holaway, \& Heimberg, 2004). In vivo exposure involves direct confrontation of actual feared stimuli, whereas imaginal exposure involves visualising feared situations (Olatunji, Cisler, \& Deacon, 2010). The client gradually confronts each situation, from least to most feared, and engages with each item until anxiety decreases (Rodebaugh et al., 2004).

There are two prevailing theoretical models related to learning that explain how exposure therapy reduces anxiety (Abramowitz, 2013). Emotional processing theory (Foa \& Kozak, 1986) and the inhibitory learning model (Craske et al., 2008) both postulate that exposure allows individuals to learn corrective information about a feared stimulus. Emotional processing theory specifically postulates that feared stimuli activate a fear structure (Foa \& Kozak, 1986). When corrective information is incompatible with this fear structure, it is replaced with a new, non-fear structure (Foa \& Kozak, 1986). However, the inhibitory learning model emphasises tolerating rather than replacing fear (Craske et al., 2008). It posits that while old, fear-based learning is not erased by extinction, new learning can inhibit its expression (Craske et al., 2008).

A large body of research supports the efficacy of in vivo and imaginal exposure treatments for reducing symptoms of social anxiety, although the methods do not work for all individuals with social anxiety (e.g., Acarturk, Cuijpers, van Straten, \& de Graaf, 2009; Feske \& Chambless, 1995; Gould, Buckminster, Pollack, Otto, \& Massachusetts, 1997; Powers, Sigmarsson, \& Emmelkamp, 2008; Turner, Beidel, \& Jacob 1994).

\section{Virtual Reality Exposure Therapy}

In recent years, exposure therapy through virtual reality has gained substantial attention as an intervention for social anxiety (Powers \& Emmelkamp, 2008; Wallach, Safir, \& Bar-Zvi, 2009). Virtual reality technology integrates computer graphics, visual displays, motion tracking and other sensory devices to give the user a multisensory and realistic experience (Bush, 2008; Krijn, Emmelkamp, Olafsson, \& Biemond, 2004; Powers \& Emmelkamp, 2008). A head-mounted display is typically used to immerse clients in the virtual environment (Krijn et al., 2004).

During virtual reality exposure therapy (VRET), the client confronts computer-generated simulations of feared situations (Anderson et al., 2013). Trained therapists conduct the treatment in a private, safe, and controlled environment (Bush, 2008). Virtual technology is programmable, and environments can be customised to clients' specific social anxieties, in accordance with their fear and avoidance hierarchy (Krijn et al., 2004; Vanni et al., 2013).

VRET may be useful in addressing the shortcomings of established methods of exposure, particularly in relation to cost-effectiveness, convenience, treatment acceptability, treatment availability, and difficulties with clients visualising scenes during imaginal exposure (Anderson et al., 2013; Choy, Fyer, \& Lipsitz, 2007; Krijn et al., 2004). Furthermore, VRET offers an appealing and novel treatment approach, and may be useful for those who show reluctance toward participating in vivo or imaginal exposure (Benedek \& Wynn, 2016; Bush, 2008). Because of these advantages, VRET might have the potential to lead individuals to seek treatment who otherwise might not do so (Powers \& Emmelkamp, 2008).

\section{The Efficacy of Virtual Reality Exposure Therapy for Social Anxiety}

To date, three meta-analyses have explored the efficacy of VRET for a range of anxiety disorders (Opriş et al., 2012; Parsons \& Rizzo, 2008; Powers \& Emmelkamp, 2008). One other meta-analysis 
(Kampmann, Emmelkamp, \& Morina, 2016) investigated the efficacy of treating social anxiety with a variety of technological interventions, including VRET.

Parsons and Rizzo (2008) investigated the pre-post effects of VRET for social phobia and reported a meta-analytic effect size of $0.96,95 \%$ CI $[0.34,1.59]$, indicating a reduction in anxiety symptoms following VRET. However, the authors only analysed results for VRET conditions and did not compare their findings to waitlist control groups or standard treatments, making it difficult to determine the relative efficacy of VRET. In contrast, Powers and Emmelkamp (2008) did make a comparison between VRET and control conditions for two social phobia studies. They reported a between-groups, meta-analytic Hedges' $g$ of 0.73 (no reported confidence interval), indicating that VRET was more efficacious than control conditions in the treatment of social anxiety. The meta-analysis combined waitlist control conditions and standard exposure control conditions in one meta-analysis, making the results hard to interpret.

More recently, Opriş et al. (2012) and Kampmann, Emmelkamp, and Morina (2016) examined the efficacy of VRET compared to waitlist controls. Opriş et al. (2012) reported a $d$ of $1.01,95 \%$ CI [0.69, 1.33] across three social phobia studies, indicating VRET outperformed waitlist on outcome measures of social anxiety. Kampmann, Emmelkamp, and Morina (2016) reported a similarly large and significant effect size across three studies, with $g=0.82,95 \%$ CI $[0.13,1.51]$. Both authors also compared VRET to standard, empirically supported treatments for social anxiety, including in vivo and imaginal exposure. Opriş et al. (2012) reported a non-significant effect size in favour of in vivo and imaginal exposure $(d=0.13,95 \%$ CI $[-0.11,0.38]$ for three social phobia studies, suggesting that VRET and standard treatments had comparable efficacy. Kampmann, Emmelkamp, and Morina (2016) reported similar non-significant findings in favour of VR in comparison to empirically supported treatments across three studies, with $g=-0.24,95 \%$ CI $[-0.71,0.23]$. A difference between these two meta-analyses was that Kampmann, Emmelkamp, and Morina (2016) restricted their analysis to randomised control trials and participants with diagnosed social anxiety disorder, whereas Opriş et al. (2012) did not require participants to have a clinical diagnosis and included studies with various methodological designs (e.g., non-random, quasi-random).

In each of these meta-analyses, researchers examined the efficacy of VRET for social anxiety by including it as a subcategory of a larger analysis. Within these subanalyses, the number of studies on social anxiety ranged from two to four, and the total number of participants ranged from 50 to 216. Moreover, the researchers included some small studies with low participant numbers. When compared to bigger studies, smaller studies often show larger and different treatment effects (Sterne, Gavaghan, \& Egger, 2000). Further, small studies are regularly selected for publication because of their statistically significant results (Sterne et al., 2000). This phenomenon contributes to publication bias (publishing only favourable results) and can therefore affect the generalisability of effect sizes in meta-analysis (Sterne et al., 2000). Although Kampmann, Emmelkamp, and Morina (2016) acknowledged the issue of publication bias in the report of their meta-analysis, they could not examine its influence on effect sizes in their VRET analysis because of the low number of studies included.

Individual studies considered in the reviewed meta-analyses investigated the efficacy of VRET for social anxiety by assessing participants' social anxiety symptomatology with various outcome measures, such as the Liebowitz Social Anxiety Scale (Liebowitz, 1987), which has high levels of reliability and validity (Heimberg et al., 1999), and behavioural measures, such as total time speaking, which have face validity.

In sum, previous meta-analytic studies of the effects of VRET for social anxiety have had two main deficiencies: a very low number of studies and a lack of evaluation of publication bias. While a meta-analysis only requires a minimum of two studies (Valentine, Pigott, \& Rothstein, 2010), increasing this number tends to enhance the generalisability of results (Schmidt \& Hunter, 2014).

\section{Aims and Hypotheses of the Present Study}

The overall objective of the present study was to examine, through two meta-analyses, the efficacy of VRET for reducing symptoms of social anxiety. We aimed to improve on prior analyses by employing 
a more methodologically rigorous design. We further aimed to consider the impact of publication bias in order to improve the precision of effect size estimates. First, we assessed the efficacy of VRET compared to a waitlist control. Second, we compared the efficacy of VRET to the standard treatments of in vivo or imaginal exposure. We tested the following hypotheses in two separate meta-analyses:

H1: Virtual reality exposure therapy would demonstrate greater efficacy in reducing social anxiety than a waitlist control condition.

$\mathrm{H} 2$ : The standard treatments of in vivo or imaginal exposure would demonstrate greater efficacy in reducing social anxiety when compared to virtual reality exposure therapy.

\section{Method}

\section{Literature Search}

We searched the electronic databases PubMed, EBSCOhost, Proquest Central, PsychINFO and Proquest Dissertations and Theses in July 2017 for published and unpublished studies. We searched the databases using Boolean operators to link the search terms and phrases 'virtual reality exposure therapy' or 'VRET' or 'virtual reality therapy' or 'VRT' and 'social anxiety' or 'social phobia'. We sought studies in Google Scholar by searching in the title using the exact phrases 'virtual reality exposure therapy' or 'virtual reality therapy', 'VRT', or 'VRET', and at least one of the terms 'social', 'anxiety', or 'phobia'. There were no date limits set on the search. Articles had to be in English. We sought further studies from reference lists of retrieved articles. We wrote to authors of studies included in the meta-analysis to enquire after unpublished studies investigating the effects of VRET on social anxiety. We evaluated studies for inclusion by systematically analysing the title and abstract and then the full text. Figure 1 provides a summary of the search process.

\section{Inclusion Criteria and Evaluation Process}

Studies had to meet the following criteria for inclusion in the meta-analysis: (1) The study must be a well-controlled trial with random assignment, quasi-random assignment or participant matching procedures that minimise bias. (2) The study must not use the same sample as another study. (3) The study report must be in English. (4) The study must have a VRET condition and at least one comparison condition, which is a waitlist or an exposure treatment using in vivo or imaginal exposure. (5) Comparison treatments must not include VRET. (6) The article must provide treatment assessment data for social anxiety symptomatology, such as appropriate reporting of means and standard deviations. (7) Social anxiety must be the main target of the intervention.

We excluded measures not relating directly to social anxiety, such as depression, general anxiety, and quality-of-life scales. Excluding these measures maintained the focus on social anxiety. We used outcome-measure totals rather than subscale values, if researchers reported both. Two studies used the Self-Statements During Public Speaking (Hofmann \& DiBartolo, 2000) scale, which contains positive and negative subscales. The negative subscale is a direct measure of social anxiety, shows more sensitivity to change than the positive scale, is more highly correlated with social anxiety, and is better able to differentiate between individuals with and without social anxiety (Hofmann \& DiBartolo, 2000). As a result, we included only the negative subscale in the analysis.

The time of final comparison for each of the two meta-analysis was the latest measured time point during which participants remained in their originally allocated conditions, unless more than half the participants were lost by that time point. If available, we used carry-forward intention-to-treat (ITT) data because it offers a more conservative estimate of treatment outcomes (Gupta, 2011). In ITT, the pretreatment scores of participants who do not complete the postassessment are carried forward to serve as their post-intervention scores. 
Figure 1. PRISMA 2009 flow diagram. Note: Format from Moher, Liberati, Tetzlaff, Altman, \& The PRISMA Group (2009).

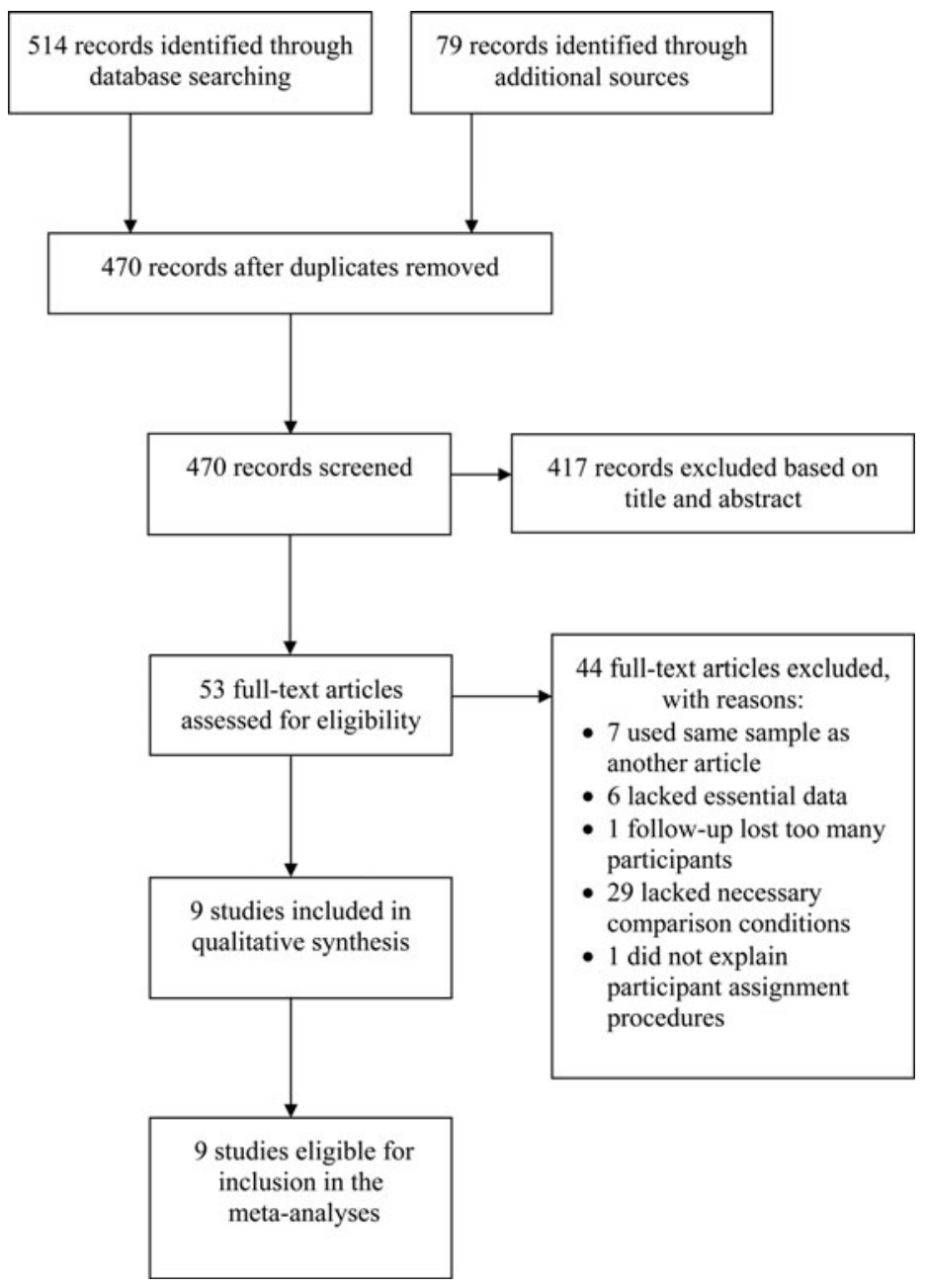

The authors Heuett and Heuett (2011) informed us, upon our contacting them, that they randomly assigned participants in their study to treatment conditions but not to the waitlist. The waitlist was comprised of individuals who filled out pretest forms incorrectly or were absent during the baseline assessment. There was evident systematic bias in the participant assignment to this condition. Therefore, we excluded the waitlist conditions from the meta-analysis. Furthermore, the data reported for two of the outcome measures at pretreatment in Heuett and Heuett's imaginal exposure condition were implausible in an absolute sense and inconsistent with the other conditions, likely due to printing errors in the article. Therefore, we excluded from the analysis the results reported for the Willingness to Communicate scale and the Self-Perceived Communication Competence scale.

The results reported in Safir, Wallach, and Bar-Zvi (2012) are the follow-up assessment for Wallach et al. (2009). We used the 1-year data in Safir et al. (2012) for the comparison of VRET and standard treatments. Since the researchers did not assess waitlist at 1 year, we used the 12 -week time point for the VRET and waitlist comparison.

We included two studies that used quasi-random assignment to condition and one study that used matching of participants prior to assignment to condition. Wallach et al. (2009) allocated participants to conditions by order of arrival, which is not true random assignment (Torgerson \& Torgerson, 2008). Further, Wallach et al. (2009) used weighted assignment later in the study due to sample 
attrition, which allowed the researchers to maintain groups of equal size. Harris, Kemmerling, and North (2002) allocated four counsellor-referred participants straight to the waitlist and randomly assigned the remaining participants. Klinger et al. (2005) matched participants on key variables (e.g., age, gender) before assignment to conditions. Despite the lack of random assignment, we considered this a well-controlled trial and included it in the meta-analysis.

\section{Study Coding and Intercoder Agreement}

Two of us jointly coded the following information for each study: publication characteristics (author and year of publication); age of participants; nature of the comparison group (treatment with exposure or waitlist); nature of participant assignment; outcome measures for assessing social anxiety symptoms; time from baseline to final comparison of conditions; number of treatment sessions; study results (sample size, pre- and postassessment means and standard deviations); and the effect direction (positive or negative).

The third author independently checked the coding by randomly selecting four studies, which included 25 lines of data (out of a total of 50 lines) and 275 entries. For continuous data entries, we considered an agreement to be a variance of anything less than $5 \%$ of the original coded value. The agreement rate was $96 \%$. Where there were disagreements, we made final decisions by consensus.

\section{Statistical Procedure and Data Analysis}

We calculated effect sizes using the coded values of the included studies, such as pre- and postassessment means and standard deviations for conditions. We used Hedges' $g$, which is closely related to Cohen's (1988) $d$, as the unbiased estimate of effect size. We used a random-effects model in the analysis to allow for the possibility that effect sizes between studies differ (Borenstein, Hedges, Higgins, \& Rothstein, 2010). The $Q$-value and $I^{2}$ assessed heterogeneity across studies. While the $Q$-value is a significance test, $I^{2}$ reports what percentage of total variability across studies in meta-analysis is due to between-study variability rather than to chance. Higgins and Thompson (2002) suggested tentative values of $I^{2}$ in which $30 \%$ is mild, and exceeding $50 \%$ is large. A 95\% confidence interval and a $p$ value were computed for each model. We assessed the impact of publication bias using Orwin's (1983) failsafe $N$, Duvall and Tweedie's (1998) trim and fill method, and by viewing funnel plots. Negative values of $g$ suggested results favoured standard treatment efficacy, whereas positive values indicated results favoured VRET efficacy. We conducted all analyses using Comprehensive Meta-Analysis (Version 3.3.070, 2014).

We first performed analyses with all studies relevant to each analysis. However, not every study used random assignment. Lacking randomisation makes these studies more susceptible to confounding bias (see Faber, Ravaud, Riveros, Perrodeau, \& Dechartres, 2016). Randomised control trials are considered the gold standard in research design and provide the best evidence for assessing the efficacy of treatment (Faraoni \& Schaefer, 2016). Further, randomised trials increase the statistical power and precision of estimated effects in meta-analysis (Wetterslev, Thorlund, Brok, \& Gluud, 2008). So, we conducted a second set of analyses including only trials using random assignment.

\section{Results}

The search strategy resulted in nine relevant studies, with a total of 573 participants. The meta-analysis data file can be obtained by contacting the corresponding author. Table 1 provides a summary of the key characteristics of the included studies. Figure 2 shows: (1) the studies used in the comparison of VRET and waitlist control conditions analysis, and (2) the studies used in the comparison of VRET and in-vivo or imaginal exposure therapy analysis and a forest plot of the effect sizes. 


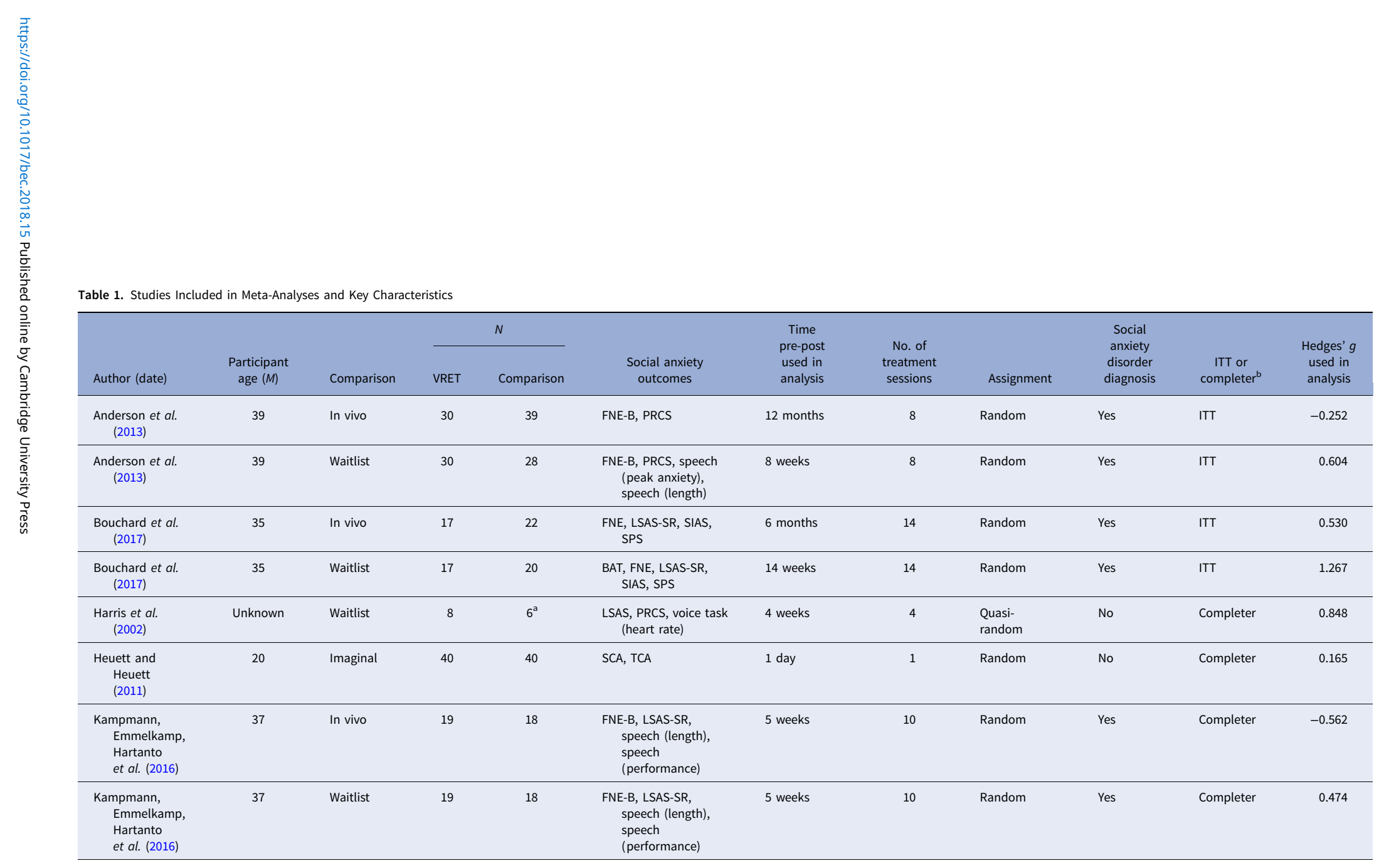




\begin{tabular}{|c|c|c|c|c|c|c|c|c|c|c|c|}
\hline $\begin{array}{l}\text { Klinger et al. } \\
\quad(2005)\end{array}$ & 32 & In vivo & 18 & 18 & RAS, CGI, LSAS, SCIA & 12 weeks & 12 & $\begin{array}{l}\text { Participant } \\
\text { matching }\end{array}$ & Yes & Completer & 0.054 \\
\hline $\begin{array}{l}\text { Robillard, } \\
\text { Bouchard, } \\
\text { Dumoulin, } \\
\text { Guitard, and } \\
\text { Klinger } \\
\text { (2010) }\end{array}$ & 35 & In vivo & 14 & 16 & $\begin{array}{l}\text { ASC-C, ASC-P, FNE, } \\
\text { LSAS, SPS }\end{array}$ & 16 weeks & 16 & Random & Yes & Completer & 0.486 \\
\hline $\begin{array}{l}\text { Robillard et al. } \\
\quad(2010)\end{array}$ & 35 & Waitlist & 14 & 15 & $\begin{array}{l}\text { ASC-C, ASC-P, FNE, } \\
\text { LSAS, SPS }\end{array}$ & 16 weeks & 16 & Random & Yes & Completer & 1.57 \\
\hline $\begin{array}{l}\text { Wallach et al. } \\
\quad(2009)\end{array}$ & 27 & Waitlist & 28 & 30 & $\begin{array}{l}\text { FNE, LSAS-Avoidance, } \\
\text { LSAS-Fear, SSPS } \\
\text { Negative }\end{array}$ & 12 weeks & 12 & Quasi-random & No & Completer & 0.57 \\
\hline $\begin{array}{l}\text { Safir et al. } \\
\text { (2012) }\end{array}$ & 27 & Imaginal & 25 & 24 & $\begin{array}{l}\text { FNE, LSAS-Avoidance, } \\
\text { LSAS-Fear, SSPS } \\
\text { Negative }\end{array}$ & 1 year & 12 & Quasi-random & No & Completer & -0.362 \\
\hline
\end{tabular}

Note: FNE-B = The Fear of Negative Evaluation - Brief Form; PRCS = Personal Report of Confidence as a Speaker; FNE = Fear of Negative Evaluation, LSAS-SR=Leibowitz Social Anxiety Scale - Self-Reported version; SIAS = Social Interaction Anxiety Scale; SPS = Social Phobia Scale; BAT = Behavioural Assessment Task; LSAS=Leibowitz Social Anxiety Scale; SCA= state communication apprehension; TCA = trait communication apprehension; RAS = Rathus Assertiveness Schedule; CGI = Clinical Global Impressions Scale; SCIA = The Questionnaire on Social Contexts Inducing Anxiety; ASC-C = Appraisal of Social Concerns consequences; ASC-P = Appraisal of Social Concerns - probability; SSPS = Self-Statements During Public Speaking; Completer = Completed post-treatment assessment.

${ }^{\mathrm{a}} n=5$ for voice task. ${ }^{\mathrm{b}} \mathrm{ITT}=$ Carry-forward intention-to-treat analysis. 
Study name

VRET compared to waitlist: all studies

Anderson et al. (2013)

Bouchard et al. (2017)

Harris et al. (2002)

Kampmann, Emmelkamp, Hartanto et al. (2016)

Robillard et al. (2010)

Wallach et al. (2009)

Overall effect size

VRET compared to waitlist: only studies with random assignment

Anderson et al. (2013)

Bouchard et al. (2017)

Kampmann, Emmelkamp, Hartanto et al. (2016)

Robillard et al. (2010)

Overall effect size

VRET compared to standard treatments: all studies

Anderson et al. (2013)

Bouchard et al. (2017)

Heuett and Heuett (2011)

Kampmann, Emmelkamp, Hartanto et al. (2016)

Klinger et al. (2005)

Robillard et al. (2010)

Safir et al. (2012)

Overall effect size

VRET compared to standard treatments: only studies with random assignment

Anderson et al. (2013)

Bouchard et al. (2017)

Heuett and Heuett (2011)

Kampmann, Emmelkamp, Hartanto et al. (2016)

Robillard et al. (2010)

Overall effect size
Hedges' $g$ and $95 \%$ CI

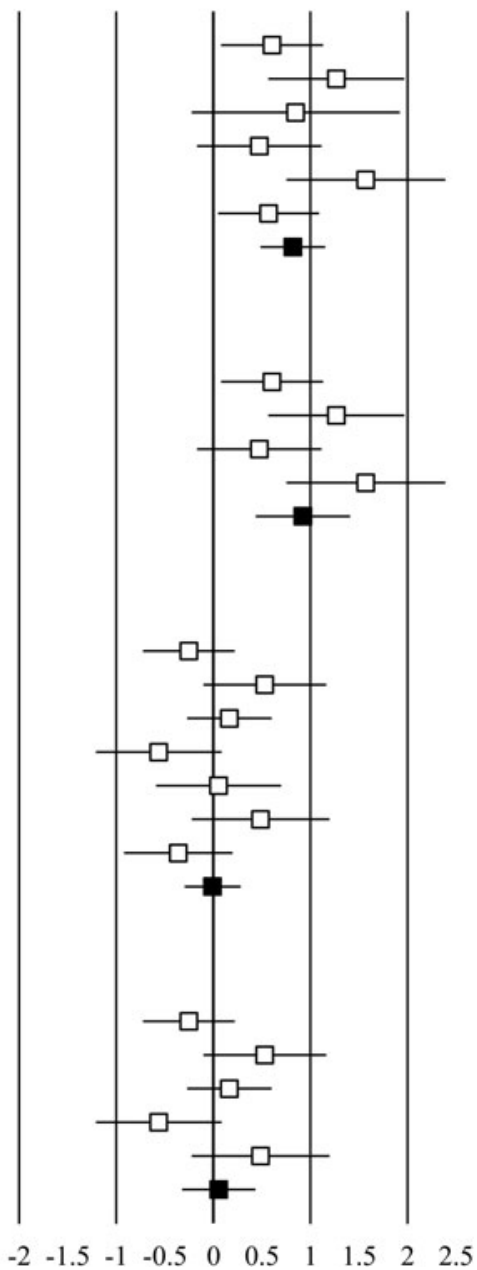

Figure 2. Studies included in each analysis and a forest plot of their effect size estimates. In the forest plot, white boxes represent the measured effect size for each study, and the confidence intervals define the precision of each estimate. Black boxes represent the overall effect summary for each analysis.

\section{Virtual Reality Exposure Therapy Compared to Waitlist}

Six studies reported results relevant to a comparison of VRET and waitlist control conditions. The overall effect size across the six studies and their 233 participants was significant, with $g=0.82$, $p<.001,95 \%$ CI $[0.49,1.15]$. This finding supported the first research hypothesis that VRET would lead to better outcomes than a waitlist control. A $Q$-value of 7.40, $d f(5), p=.193$, indicated no significant heterogeneity across the studies. Hence, moderator analyses would be inappropriate. The $I^{2}$ value of 32.40 suggested that $32 \%$ of variance was due to heterogeneity across studies rather than to chance.

The funnel plot (Figure 3) showed moderate asymmetry of effect sizes for the six studies, suggesting potential publication bias. Duvall and Tweedie's (1998) trim and fill also indicated publication bias and corrected for this by adding one missing study to the plot, leading to an adjusted effect size of $g=0.71,95 \%$ CI $[0.34,1.09]$. Orwin's fail-safe $N$ indicated that it would take 18 missing studies with $g=0$ to bring the overall effect size down to a trivial level (trivial $g=0.2$ ). 


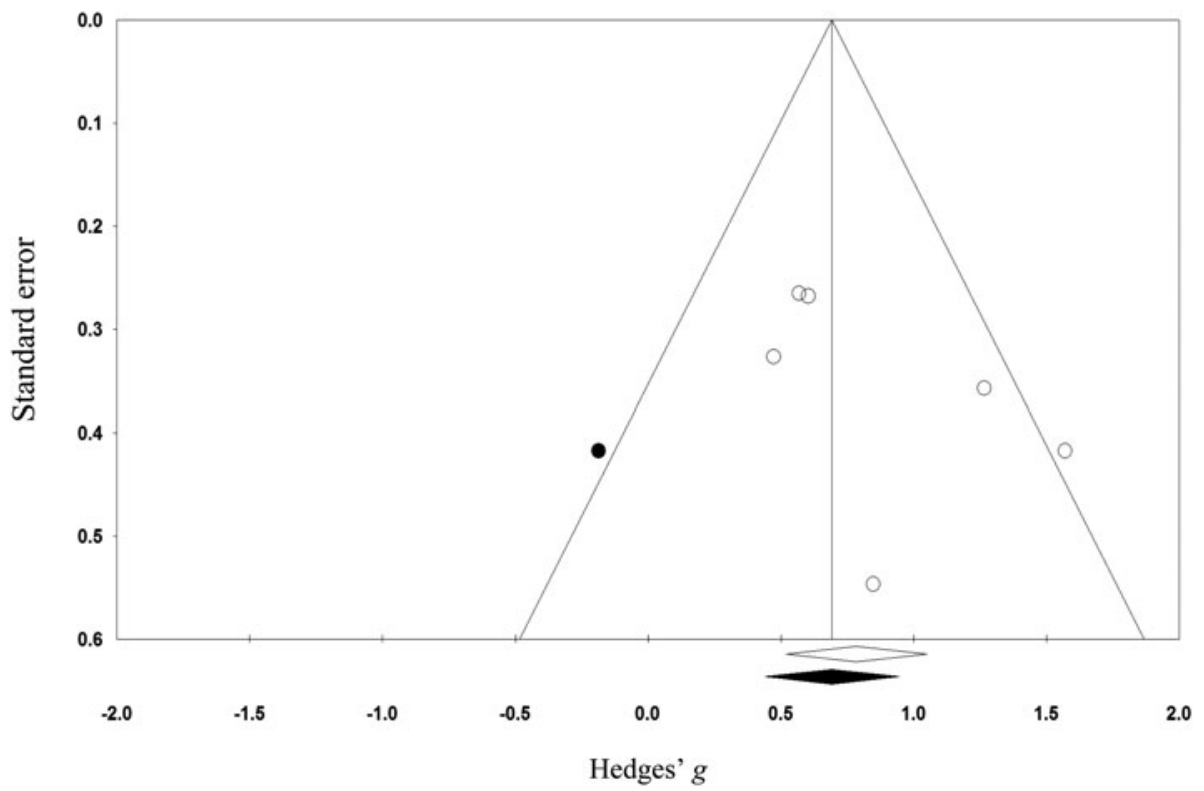

Figure 3. Funnel plot showing study effect sizes and standard error. White circles represent the six studies included in the analysis. The black circle shows the imputed study.

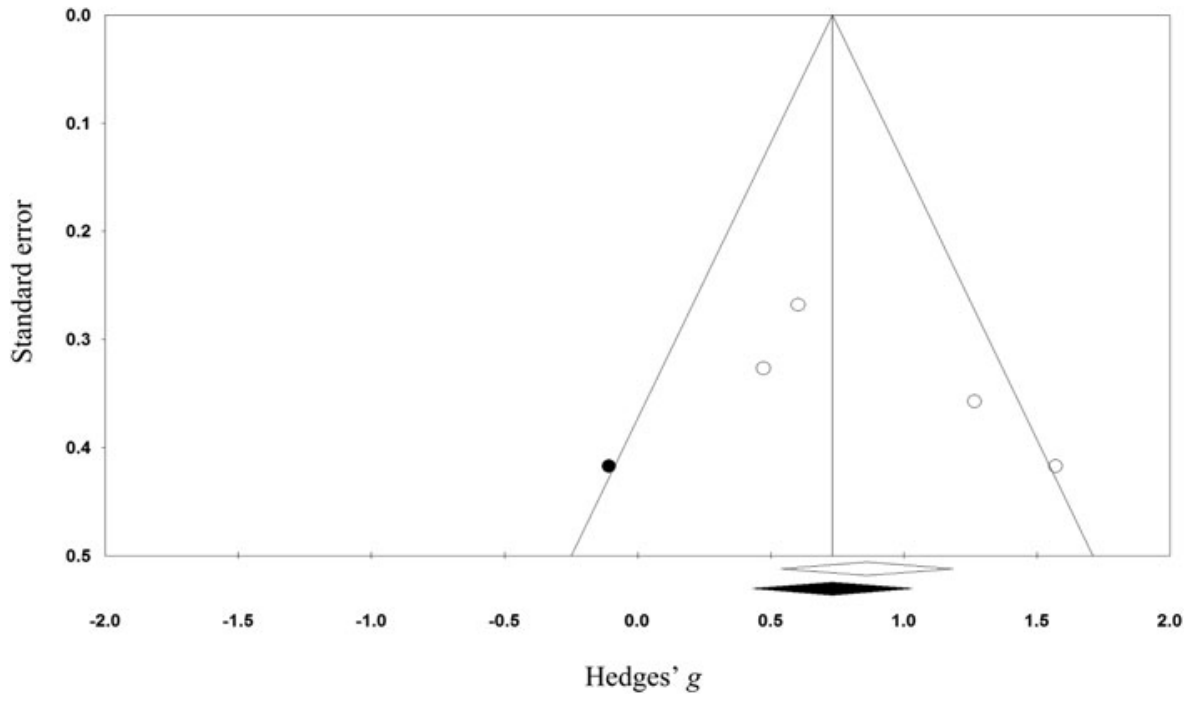

Figure 4. Funnel plot of effect sizes and standard error in the analysis of studies using random assignment. White circles represent included studies. The black circle shows the imputed study.

With the analysis limited to the four studies with random assignment, the overall effect size across a total of 161 participants was significant, with $g=0.92, p<.001,95 \%$ CI $[0.44,1.41]$. A $Q$-value of 6.51 , $d f(3), p=.089$, indicated that the set of studies were not significantly heterogeneous, $I^{2}=53.90$.

With only studies that used random assignment, the funnel plot showed minor to moderate asymmetry, suggesting some publication bias (Figure 4). Duvall and Tweedie's (1998) trim and fill indicated bias and imputed one missing study into the funnel plot. Taking this bias into account, the resulting 
effect size fell to $g=0.76,95 \%$ CI $[0.25,1.27]$. Orwin's fail-safe $N$ indicated that it would take 14 missing studies with $g=0$ to bring the overall effect size down to a trivial level (trivial $g=0.2$ ).

\section{Virtual Reality Exposure Therapy Compared to Standard Treatments}

There were seven studies and a total of 340 participants included in the analysis of all studies comparing VRET to in vivo or imaginal exposure. The overall effect was in the direction of standard treatments; however, the effect size was extremely small and non-significant, with $g=-0.01, p=.955$, $95 \%$ CI $[-0.30,0.28]$. A $Q$-value of $10.68, d f(6), p=.099, I^{2}=43.83$, indicated no significant heterogeneity across the studies. Hence, moderator analyses would be inappropriate.

When only considering studies with random assignment, the overall effect across five studies and 255 participants was in favour of VRET, with $g=0.06, p=.770,95 \%$ CI $[-0.32,0.43]$. The studies were not significantly heterogeneous, $Q=8.95, p=.062, I^{2}=55.31$.

There were similar results in publication bias analyses for the seven studies and for the five studies that used random assignment. There was no asymmetry detected in the funnel plots of effect sizes. Further, Duvall and Tweedie's (1998) trim and fill did not impute any missing studies. Orwin's failsafe $N$ analysis was not useful because the Hedges' $g$ values were already in the trivial category.

\section{Discussion}

Nine well-controlled trials provided information about the efficacy of VRET for treating social anxiety. The results of the first meta-analysis, consisting of six studies and 233 participants, showed a medium-to-large, significant overall effect size, indicating that VRET was more efficacious than waitlist. This finding provided support for the first hypothesis, that VRET is efficacious. Results of the second meta-analysis, consisting of seven studies and 340 participants, showed no significant differences in effect sizes when comparing VRET to in vivo or imaginal exposure. This finding did not support the second hypothesis, which was that the usual exposure treatment would be more efficacious than VR exposure treatment. Analyses including only trials with random assignment produced similar results for both hypotheses.

\section{Virtual Reality Exposure Therapy Compared to Waitlist}

The results indicated that VRET is superior to waitlist conditions in reducing symptoms of social anxiety. This finding was consistent with smaller past meta-analyses by Opriş et al. (2012) and Kampmann, Emmelkamp, and Morina (2016). The present results indicate that VRET tends to reduce symptoms of social anxiety. Further, the findings provide support for the extinction aspect of learning theory, for the corrective learning element of emotional processing theory (Foa \& Kozak, 1986), and for the inhibitory learning model (Craske et al., 2008).

This study was the first to address publication bias when considering VRET for social anxiety. In the comparison between VRET and waitlist, results showed that effect sizes went from large to medium when taking this bias into account, suggesting there was some initial overestimation of the overall effect size. A lack of investigation into publication bias in previous research raises questions about the validity of their findings, and the adjusted effect size of the present meta-analysis could be a more accurate reflection of the effect. Clinicians frequently use meta-analyses as a resource to determine best treatment options for clients (Petitti, 2000), and the present study's findings may provide a more methodologically sound basis for informing treatment decisions.

\section{Virtual Reality Exposure Therapy Compared to Standard Treatments}

The results obtained from this set of studies showed no significant difference in effect sizes between VRET and in vivo or imaginal exposure. This finding is in line with results of the meta-analyses of Kampmann, 
Emmelkamp, and Morina (2016) and Opriş et al. (2012). While the results suggest that there is no significant advantage to using one treatment approach over another, the non-significant difference between these two conditions does not by itself prove a lack of difference. Rather, it indicates that studies to date do not collectively show that VRET is less effective than standard treatments for social anxiety.

The fact that the second hypothesis was not supported might be explained by the 'dodo bird conjecture', which postulates that when bona fide psychotherapies are compared, they produce equivalent outcomes (Wampold et al., 1997). Moreover, Wampold et al. (1997) reported smaller differences in effect when comparing treatments belonging to the same conceptual category of treatment, than to treatments belonging to dissimilar categories. Considering in vivo exposure, imaginal exposure, and VRET contain common features inherent to the category of exposure-based behavioural interventions (Oskam, 2005), similarity of treatment effects found in the present meta-analysis is in line with Wampold's (1997) findings. Common factors could also explain the negligible difference between these exposure therapies (Drisko, 2004). Common factor models posit that if psychotherapies are equally efficacious, it is due to essential ingredients that they all share (e.g., therapeutic alliance, placebo effects) rather than the unique elements of the therapy (Drisko, 2004; Imel \& Wampold, 2008).

The results of the present two meta-analyses indicate that VR exposure treatment tends to be efficacious, with no significant evidence that it leads to less benefit than the usual treatments of in vivo and imaginal exposure. These results may be useful for clients and clinicians seeking evidence to inform treatment decisions.

The present results add to prior meta-analyses of the efficacy of VRET for social anxiety by: (1) including about double the number of studies, (2) adjusting for publication bias, and (3) reporting results for both RCTs and for all well-controlled studies. These advantages make the results more reliable and generalisable.

\section{Study Limitations}

Despite including more studies than previous investigations, this analysis was still based on a small number of studies. It is possible, despite our comprehensive search process, that additional studies exist that are relevant to the present meta-analysis. Including the results might lead to different results. However, Orwin's failsafe $N$ indicates that many studies with no effect would have to be included to change the conclusion about the effects of VRET compared to no treatment.

\section{Future Directions}

The potential benefits of VRET signify a need for additional high-quality trials investigating its optimal application as an intervention for social anxiety. Adding more studies with increased methodological rigour to the existing body of research would assist with improving the generalisability of findings. Researchers could maximise the usefulness of future studies by conducting randomised control trials, using large sample sizes, including follow-up data, and using well-validated measures of social anxiety outcomes. Increasingly realistic and multifaceted virtual reality technology offers the opportunity to research how to maximise the beneficial use of virtual reality in reducing social anxiety.

VR exposure treatment has potential advantages over the usual exposure in vivo or in imagery. These potential advantages include greater acceptability, along with easier provision for some clients of varied social situations than is possible in vivo or in imagery. In the future, researchers could compare the willingness of socially anxious individuals to seek or to accept VRET compared to other types of exposure treatments. This matter is important because so few socially anxious individuals ever seek treatment (Crome et al., 2015; Fehm et al., 2005). Finally, researchers could evaluate the effects of VR exposure without therapist involvement. Such intervention, possibly delivered through inexpensive mobile phone applications and using inexpensive VR hardware, could provide a new, valuable option to individuals who are too socially anxious to seek professional help or who live far from expert therapists. 


\section{References}

References marked with an asterisk indicate studies included in the meta-analysis.

Abramowitz JS (2013) The practice of exposure therapy: Relevance of cognitive-behavioral theory and extinction theory. Behavior Therapy 44, 548-558. https://doi.org/10.1016/j.beth.2013.03.003

Acarturk C, Cuijpers P, van Straten A and de Graaf R (2009) Psychological treatment of social anxiety disorder: A meta-analysis. Psychological Medicine 39, 241-254. https://doi.org/10.1017/S0033291708003590

American Psychiatric Association. (2013) Diagnostic and statistical manual of mental disorders, 5th Ed. Washington, DC: Author.

${ }^{*}$ Anderson PL, Price M, Edwards SM, Obasaju MA, Schmertz SK, Zimand E and Calamaras MR (2013) Virtual reality exposure therapy for social anxiety disorder: A randomized controlled trial. Journal of Consulting and Clinical Psychology 81, 751-760. https://doi.org/10.1037/a0033559

Benedek DM and Wynn GH (2016) Complementary and alternative medicine for PTSD. New York, NY: Oxford University Press.

Borenstein M, Hedges LV, Higgins JPT and Rothstein HR (2010) A basic introduction to fixed-effect and random-effects models for meta-analysis. Research Synthesis Methods 1, 97-111. https://doi.org/10.1002/jrsm.12

${ }^{*}$ Bouchard S, Dumoulin S, Robillard G, Guitard T, Klinger É., Forget H, ... Roucaut FX (2017) Virtual reality compared with in vivo exposure in the treatment of social anxiety disorder: A three-arm randomised controlled trial. The British Journal of Psychiatry 210, 276-283. https://doi.org/10.1192/bjp.bp.116.184234

Bush J (2008) Viability of virtual reality exposure therapy as a treatment alternative. Computers in Human Behavior 24, 1032 1040. https://doi.org/10.1016/j.chb.2007.03.006

Choy Y, Fyer AJ and Lipsitz JD (2007) Treatment of specific phobia in adults. Clinical Psychology Review 27, 266-286. https://doi.org/10.1016/j.cpr.2006.10.002

Cohen J (1988) Statistical power analysis for the behavioral sciences, 2nd Ed. Hillsdale, NJ: Lawrence Erlbaum Associates.

Comprehensive Meta-Analysis (Version 3.3.070). [Computer software]. Englewood, NJ: Biostat.

Craske MG, Kircanski K, Zelikowsky M, Mystkowski J, Chowdhury N and Baker A (2008) Optimizing inhibitory learning during exposure therapy. Behaviour Research and Therapy 46, 5-27. https://doi.org/10.1016/j.brat.2007.10.003

Crome E, Grove R, Baillie AJ, Sunderland M, Teesson M and Slade T (2015) DSM-IV and DSM-5 social anxiety disorder in the Australian community. Australian \& New Zealand Journal of Psychiatry 49, 227-235. https://doi:10.1177/ 0004867414546699

Deacon BJ and Abramowitz JS (2004) Cognitive and behavioral treatments for anxiety disorders: A review of meta-analytic findings. Journal of Clinical Psychology 60, 429-441. https://doi.org/10.1002/jclp.10255

Drisko JW (2004) Common factors in psychotherapy outcome: Meta-analytic findings and their implications for practice and research. Families in Society 85, 81-90. https://doi.org/10.1606/1044-3894.239

Duval S and Tweedie R (1998) Practical estimates of the effect of publication bias in meta-analysis. Australasian Epidemiologist 5, 14-17. Retrieved from https://pdfs.semanticscholar.org/bd69/631154a14fd6e4eb0fefbf4602b8falf8996. pdf

Faber T, Ravaud P, Riveros C, Perrodeau E and Dechartres A (2016) Meta-analyses including non-randomized studies of therapeutic interventions: A methodological review. BMC Medical Research Methodology 16, 35-59. https://doi.org/10. 1186/s12874-016-0136-0

Faraoni D and Schaefer ST (2016) Randomized controlled trials vs. observational studies: Why not just live together? BMC Anesthesiology 16, 102-105. https://doi.org/10.1186/s12871-016-0265-3

Fehm L, Pelissolo A, Furmark T and Wittchen H-U (2005) Size and burden of social phobia in Europe. European Neuropsychopharmacology 15, 453-462. https://doi.org/10.1016/j.euroneuro.2005.04.002

Feske U and Chambless DL (1995) Cognitive behavioral versus exposure only treatment for social phobia: A meta-analysis. Behavior Therapy 26, 695-720. https://doi.org/10.1016/S0005-7894(05)80040-1

Foa EB and Kozak MJ (1986) Emotional processing of fear: Exposure to corrective information. Psychological Bulletin 99, 20-35. https://doi.org/10.1037/0033-2909.99.1.20

Furmark T (2002) Social phobia: Overview of community surveys. Acta Psychiatrica Scandinavica 105, 84-93. https://doi. org/10.1034/j.1600-0447.2002.1r103.x

Gould RA, Buckminster S, Pollack MH, Otto MW and Massachusetts LY (1997) Cognitive-behavioral and pharmacological treatment for social phobia: A meta-analysis. Clinical Psychology: Science and Practice 4, 291-306. https://doi. org/10.1111/j.1468-2850.1997.tb00123.x

Gupta SK (2011) Intention-to-treat concept: A review. Perspectives in Clinical Research 2, 109-112. https://doi.org/10.4103/ 2229-3485.83221

${ }^{*}$ Harris SR, Kemmerling RL and North MM (2002) Brief virtual reality therapy for public speaking anxiety. Cyberpsychology \& Behavior 5, 543-550. https://doi.org/10.1089/109493102321018187

Heimberg RG (2002) Cognitive-behavioral therapy for social anxiety disorder: Current status and future directions. Biological Psychiatry 51, 101-108. https://doi.org/10.1016/S0006-3223(01)01183-0. 
Heimberg RG, Horner KJ, Juster HR, Safren SA, Brown EJ, Schneier FR and Liebowitz MR (1999) Psychometric properties of the Liebowitz Social Anxiety Scale. Psychological Medicine 29, 199-212. Retrieved from https://www.ncbi.nlm.nih. gov/pubmed/10077308

${ }^{\star}$ Heuett BL and Heuett KB (2011) Virtual reality therapy: A means of reducing public speaking anxiety. International Journal of Humanities and Social Science 1, 1-6. Retrieved from https://pdfs.semanticscholar.org/61df/ef3a4ed1b76e3e0aa8e7660535ba4b617adb.pdf

Higgins JPT and Thompson SG (2002) Quantifying heterogeneity in a meta-analysis. Statistics in Medicine 21, $1539-1558$. https://doi.org/10.1002/sim.1186

Hofmann SG and DiBartolo PM (2000) An instrument to assess self-statements during public speaking: Scale development and preliminary psychometric properties. Behavior Therapy 31, 499-515. https://doi.org/10.1016/S0005-7894(00)80027-1

Hofmann SG and DiBartolo PM (2014) Social anxiety clinical, developmental, and social perspectives, 3rd Ed. San Diego, CA: Academic Press.

Hofmann SG, Heinrichs N and Moscovitch DA (2004) The nature and expression of social phobia: Toward a new classification. Clinical Psychology Review 24, 769-797. https://doi.org/10.1016/j.cpr.2004.07.004

Imel $\mathbf{Z}$ and Wampold BE (2008) The importance of treatment and the science of common factors in psychotherapy. In Brown SD \& Lent RW (eds), Handbook of counseling psychology, 4th Ed. Hoboken, NJ: John Wiley \& Sons, pp. 249-266

*Kampmann IL, Emmelkamp PMG, Hartanto D, Brinkman WP, Zijlstra BJ and Morina N (2016) Exposure to virtual social interactions in the treatment of social anxiety disorder: A randomized controlled trial. Behaviour Research and Therapy 77, 147-156. https://doi.org/10.1016/j.brat.2015.12.016

Kampmann IL, Emmelkamp PMG and Morina N (2016) Meta-analysis of technology-assisted interventions for social anxiety disorder. Journal of Anxiety Disorders 42, 71-84. https://doi.org/10.1016/j.janxdis.2016.06.007

${ }^{*}$ Klinger E, Bouchard S, Légeron P, Roy S, Lauer F, Chemin I and Nugues P (2005) Virtual reality therapy versus cognitive behavior therapy for social phobia: A preliminary controlled study. Cyberpsychology \& Behavior 8, 76-88. https://doi.org/ 10.1089/cpb.2005.8.76

Krijn M, Emmelkamp PMG, Olafsson RP and Biemond R (2004) Virtual reality exposure therapy of anxiety disorders: A review. Clinical Psychology Review 24, 259-281. https://doi.org/10.1016/j.cpr.2004.04.001

Liebowitz MR (1987) Social phobia. Modern Problems in Pharmacopsychiatry 22, 141-173. https://oi.org/10.1159/ 000414022

Moher D, Liberati A, Tetzlaff J, Altman DG and The PRISMA Group. (2009) Preferred reporting items for systematic reviews and meta-analyses: The PRISMA Statement. PLoS Medicine 6, e1000097.

Olatunji BO, Cisler JM and Deacon BJ (2010) Efficacy of cognitive behavioral therapy for anxiety disorders: A review of meta-analytic findings. Psychiatric Clinics of North America 33, 557-577. https://doi.org/10.1016/j.psc.2010.04.002

Opriş D, Pintea S, García-Palacios A, Botella C, Szamosközi Ş. and David D (2012) Virtual reality exposure therapy in anxiety disorders: A quantitative meta-analysis. Depression and Anxiety 29, 85-93. https://doi.org/10.1002/da.20910

Orwin RG (1983) A fail-safe N for effect size in meta-analysis. Journal of Educational Statistics 8, 157-159. https://doi.org/10. 3102/10769986008002157

Oskam P (2005) Virtual reality exposure therapy (VRET) effectiveness and improvement. Paper presented at the 2 nd Twente University Student Conference on IT, Enschede, The Netherlands.

Parsons TD and Rizzo AA (2008) Affective outcomes of virtual reality exposure therapy for anxiety and specific phobias: A meta-analysis. Journal of Behavior Therapy and Experimental Psychiatry 39, 250-261. https://doi.org/10.1016/j.jbtep.2007. 07.007

Petitti DB (2000) Meta-analysis, decision analysis, and cost-effectiveness analysis: Methods for quantitative synthesis in medicine. New York, NY: Oxford University Press.

Pilling S, Mayo-Wilson E, Mavranezouli I, Kew K, Taylor C and Clark DM (2013) Recognition, assessment and treatment of social anxiety disorder: Summary of NICE guidance. British Medical Journal 346, 1-3. https://doi.org/10.1136/bmj. f2541

Powers MB and Emmelkamp PMG (2008) Virtual reality exposure therapy for anxiety disorders: A meta-analysis. Journal of Anxiety Disorders 22, 561-569. https://doi.org/10.1016/j.janxdis.2007.04.006

Powers MB, Sigmarsson SR and Emmelkamp PMG (2008) A meta-analytic review of psychological treatments for social anxiety disorder. International Journal of Cognitive Therapy 1, 94-113. https://doi.org/10.1521/ijct.2008.1.2.94

${ }^{*}$ Robillard G, Bouchard S, Dumoulin S, Guitard T and Klinger E (2010) Using virtual humans to alleviate social anxiety: Preliminary report from a comparative outcome study. Studies in Health Technology and Informatics 154, 57-60. https:// doi.org/10.3233/978-1-60750-561-7-57

Rodebaugh TL, Holaway RM and Heimberg RG (2004) The treatment of social anxiety disorder. Clinical Psychology Review 24, 883-908. https://doi.org/10.1016/j.cpr.2004.07.007

Ruscio AM (2010) The latent structure of social anxiety disorder: Consequences of shifting to a dimensional diagnosis. Journal of Abnormal Psychology 119, 662-671. https://doi.org/10.1037/a0019341 
Ruscio AM, Brown TA, Chiu WT, Sareen J, Stein MB and Kessler RC (2008) Social fears and social phobia in the United States: Results from the National Comorbidity Survey Replication. Psychological Medicine 38, 15-28. https://doi. org/10.1017/S0033291707001699

*Safir MP, Wallach HS and Bar-Zvi M (2012) Virtual reality cognitive-behavior therapy for public speaking anxiety: One-year follow-up. Behavior Modification 36, 235-246. https://doi.org/10.1177/0145445511429999

Schmidt FL and Hunter JE (2014) Methods of meta-analysis: Correcting error and bias in research findings, 3rd Ed. Thousand Oaks, CA: Sage Publications.

Sterne JAC, Gavaghan D and Egger M (2000) Publication and related bias in meta-analysis: Power of statistical tests and prevalence in the literature. Journal of Clinical Epidemiology 53, 1119-1129. https://doi.org/10.1016/S0895-4356(00) 00242-0

Torgerson DJ and Torgerson CJ (2008) Designing randomised trials in health, education and the social sciences: An introduction. New York, NY: Palgrave Macmillan.

Turner SM, Beidel DC and Jacob RG (1994) Social phobia: A comparison of behavior therapy and atenolol. Journal of Consulting and Clinical Psychology 62, 350-358. https://doi.org/10.1037/0022-006X.62.2.350

Valentine JC, Pigott TD and Rothstein HR (2010) How many studies do you need? A primer on statistical power for meta-analysis. Journal of Educational and Behavioral Statistics 35, 215-247. https://doi.org/10.3102/1076998609346961

Vanni F, Conversano C, Del Debbio A, Landi P, Carlini M, Fanciullacci C, ... Dell'Osso L (2013) A survey on virtual environment applications to fear of public speaking. European Review for Medical and Pharmacological Sciences 17, 1561-1568. Retrieved from http://www.europeanreview.org/wp/wp-content/uploads/1561-15681.pdf

${ }^{*}$ Wallach HS, Safir MP and Bar-Zvi M (2009) Virtual reality cognitive behavior therapy for public speaking anxiety. Behavior Modification 33, 314-338. https://doi.org/10.1177/0145445509331926

Wampold BE, Mondin GW, Moody M, Stich F, Benson K and Ahn H (1997) A meta-analysis of outcome studies comparing bona fide psychotherapies: Empiricially, 'all must have prizes'. Psychological Bulletin 122, 203-215. https://doi.org/ 10.1037/0033-2909.122.3.203

Wetterslev J, Thorlund K, Brok J and Gluud C (2008) Trial sequential analysis may establish when firm evidence is reached in cumulative meta-analysis. Journal of Clinical Epidemiology 61, 64-75. https://doi.org/10.1016/j.jclinepi.2007.03.013

Cite this article: Chesham RK, Malouff JM, Schutte NS (2018). Meta-Analysis of the Efficacy of Virtual Reality Exposure Therapy for Social Anxiety. Behaviour Change 35, 152-166. https://doi.org/10.1017/bec.2018.15 